\title{
Wavelet Neural Network Structure Optimization Method Based on Grey Relational-Sensitivity
}

\author{
Shiying Pan ${ }^{1, a}$, Xiuqing Wang ${ }^{2, b}$ \\ Department of Professional Technology College, Hebei Normal University, Shijiazhuang, 050024, \\ China \\ aemail: lylglg@163.com, bemail:kxu2012@163.com
}

NSFC 61175059

Keywords: Multivariate Time Series; Grey Relational-Sensitivity; Hidden Layer Node Number; Wavelet Neural Network

\begin{abstract}
In view of the wavelet neural network input number to determine the lack of theoretical guidance, the number of hidden layer nodes to determine the defects of difficult, put forward a kind of based on multivariate time series and grey relational - sensitivity of the wavelet neural network structure optimization method. This method firstly before the wavelet neural network learning, number of multivariate time series is used to determine the network's input, and then using grey relation in the process of learning - sensitivity pruning method to determine the neural network hidden layer node number, to achieve the goal of structural optimization. Through the model simulation results in the short-term wind power prediction, the results show that the method the optimized wavelet neural network to improve the wind power prediction accuracy and verified the effectiveness and feasibility of this structure optimization method, for the determination of wavelet neural network structure provides reference.
\end{abstract}

\section{Introduction}

Wavelet Neural Network(WNN) is Zhang and Benveniste presented for the first time in 1992 as a kind of wavelet analysis technique was applied to the new neural network model of artificial neural network. It is a good approximation properties of wavelet transform and artificial neural network generalization ability and self-study ability, and the nonlinear approximation properties, which is widely used in signal and image processing, pattern recognition, fault diagnosis and control, etc.

This paper presents a grey relational - sensitivity based on wavelet neural network structure optimization method. Before the network learning, determine the input the number of the network, the network learning process using grey relational - sensitivity pruning method to determine the neural network hidden layer node number, achieve the goal of structural optimization, and the optimized network is applied to wind power prediction.

\section{The Wavelet Neural Network}

Wavelet neural network is the product of combining wavelet analysis and artificial neural network, according to its structure is divided into two categories: the network of loose and tight type. Loose network based on wavelet analysis, as the pre processing means of conventional neural networks for neural network input feature vectors. Compact type is wavelet function instead of conventional neural network hidden layer effect function, respectively using wavelet function coefficient of scale and translation for the instead of the conventional network input layer to hidden layer weights and thresholds.

In this paper, nonlinear wavelet function to replace the BP neural network in the Sigmod function as a hidden layer of neural network function, constitute the wavelet neural network, the network structure as shown in figure 1, consists of input layer, hidden layer and output layer, the network nodes are connected by a weight between each layer, the same layer between the nodes are 
independent of each other, there is no connection to each other.

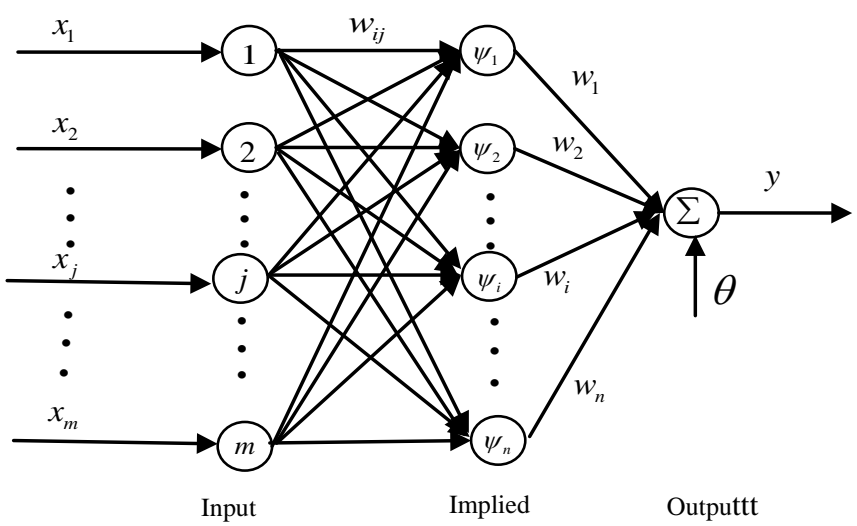

Fig.1 wavelet neural network structure

In diagram, wavelet neural network's input layer nodes for $\mathrm{m}$, output layer node number is 1 , determined by the actual application situation. Number of hidden layer nodes is $\mathrm{n}$, generally according to empirical formula.

$x_{j}$ is the first $\mathrm{j}$ a input for the network. $\mathrm{Y}$ is the output of the network. $w_{i j}$ is the input node weights between hidden nodes I and $\mathrm{j} . w_{i}$ is the hidden node i weights between hidden and output node. $\theta$ is the output node threshold. $\psi_{i}\left(a_{i}, b_{i}\right)$ is the function of the hidden node to the output node I function. $a_{i}$ and $b_{i}$ is the first $\mathrm{i}$ a hidden node scale factor and shift factor respectively . The output of wavelet neural network as follows:

$$
y=f\left(\sum_{i=1}^{n} w_{i} \psi_{i}\left(\left(\sum_{j=1}^{m} w_{i j} x_{j}-b_{i}\right) / a_{i}\right)-\theta\right)
$$

$\mathrm{f}(\bullet)$ is the function of the hidden layer to output layer functions, commonly used for the linear function. Wavelet analysis is the precondition of choosing the appropriate wavelet function function, this article selects the wavelet function Morlet which commonly used in engineering, its form is

$$
\psi(t)=\cos (1.75 t) \exp \left(-t^{2} / 2\right)
$$

Using the error gradient descent method one by one, from the output layer to input layer to adjust network parameters, after the adjustment parameters are:

$$
\begin{gathered}
w_{i}(k+1)=w_{i}(k)+\eta \sum_{p=1}^{N}\left(d^{p}-y^{p}\right) o_{i}^{p} \\
\theta(k+1)=\theta(k)+-\eta \sum_{p=1}^{N}\left(d^{p}-y^{p}\right) \\
w_{i j}(k+1)=w_{i j}(k)+\eta \sum_{p=1}^{N}\left(d^{p}-y^{p}\right) w_{i} \psi^{\prime}\left(\left(n e t_{i}^{p}-b_{i}\right) / a_{i}\right) x_{j}^{p} / a_{i}
\end{gathered}
$$

$\mathrm{j}=1,2, \ldots, \mathrm{m}$ is the number of input layer nodes. $\mathrm{i}=1,2, \ldots, \mathrm{n}$ is the number of hidden layer nodes. $\mathrm{P}$ is sample number. $\mathrm{K}$ is the number of iterations. $\eta$ is Vector $\cdot o_{i}^{p}$ is the output of a hidden node I .

$$
\operatorname{net}_{i}^{p}=\sum_{j=1}^{m} w_{i j} x_{j}^{p}
$$

\section{Determine the Wavelet Neural Network Input}

Neural network's input generally according to the actual need for sure, has a great deal of subjectivity. Too little and the neural network input, will reduce the network learning accuracy; Too much of the neural network input, will increase the learning time of the network. So in this paper, 
wavelet neural network is adopted to wind power prediction to verify the performance of the proposed method can therefore be set sequence $\{\mathrm{Yt}\}$ in response to wind power, wind speed, wind direction sequences and temperature sequence for the input sequence $\{x i t\}(I=1,2,3)$, the input sequence and ARIMAX model between response sequence is as follows:

$$
\left\{\begin{array}{l}
Y_{t}=\mu+\frac{\Theta_{1}(B)}{\Phi_{1}(B)} B^{l_{1}} x_{t}+\frac{\Theta_{2}(B)}{\Phi_{2}(B)} B^{l_{2}} x_{2 t}+\frac{\Theta_{3}(B)}{\Phi_{3}(B)} B^{l_{3}} x_{3 t}+\varepsilon_{t} \\
\varepsilon_{t}=\frac{\Theta_{0}(B)}{\Phi_{0}(B)} a_{t}
\end{array}\right.
$$

When determining the input of neural network, the first for the power sequence of wind speed, wind direction, temperature and stationarity test. Finally to white noise test of regression residual sequence, determine the number of input variables and wavelet neural network.

\section{Based on the Grey Relation-the Sensitivity of the Hidden Layer Structure of Wavelet Neural Network Optimization}

Grey relation method is according to the development trend of similar or dissimilar degree between factors (that is, the grey correlation degree) to measure the relationship between factors is a method of closely.

Suppose there are $\mathrm{N}$ the training sample, $\boldsymbol{o}_{\boldsymbol{i}}=\left(o_{i}^{1}, o_{i}^{2}, \cdots, o_{i}^{p}, \cdots, o_{i}^{N}\right)(\mathrm{I}=1,2, \ldots, \mathrm{N})$ for the wavelet neural network hidden layer of the ith node study $\mathrm{N}$ sample data output; For the output layer nodes in $\mathrm{N}$ learning sample data output. $y=\left(y^{1}, y^{2}, \cdots, y^{p}, \cdots, y^{N}\right)$ When after completing a learning network, the neural network output sequence of the expected value y mother (sequence) as reference sequence, the output sequences of a hidden node $\boldsymbol{o}_{i}$ as ” $i$ " compare sequence (sequence).

The hidden nodes "i" of grey correlation degree is defined that:

$$
r_{i}=\frac{1}{N} \sum_{p=1}^{N} \xi_{i}(p) \quad(i=1,2, \cdots, n)
$$

The size of the grey correlation degree showed that the output of the hidden node I to the influence of network output value change ability. Grey correlation degree is smaller, the hidden node I to the influence of the network output is smaller, so that the hidden nodes is redundant, you can delete.

In the process of the early stage of the wavelet neural network learning, first using the method of grey relation to "delete" thick of hidden layer nodes, until the rest of the nodes of grey correlation degree is greater than the gray correlation threshold, and then by using the sensitivity pruning method to further optimize hidden layer structure.

\section{Conclusion}

In this paper, based on the study of the wavelet neural network, in the lack of theoretical guidance to determine the number of input and hidden layer node number determine the defects of difficult, put forward a kind of grey relational - sensitivity of the wavelet neural network structure optimization method. Before the wavelet neural network learning, determine the number of input, then using grey relation in the process of learning - sensitivity pruning method to determine the neural network hidden layer node number, finally determine the structure of wavelet neural network. This article is about the structure of wavelet neural network optimization method was studied, and its learning algorithm is still adopts the traditional the steepest descent method. But the steepest descent method has slow convergence speed, easy to fall into local minimum value, therefore, later can on the learning algorithm of wavelet neural network is optimized. 


\section{References}

[1] Zhang Q, Benveniste A. Wavelet network [ J ] .IEEE Transactions on Neural Networks, 1992, 3( 6) : $889 \sim 898$.

[2] Cybeko G. Approximations by superpositions of a sigmoidal function[J]. Math Control Signals Systems, 1989,(2):303-314.

[3] M. C. Mozer, P. Smolensky. Skeletonization: A Technique for Trimming the Fat from a Network via Relevance Assessment[M]. Advances in Neural Information Processing Systems, Morgan Kaufmann, 1989:107-115. 